\title{
Avaliação do risco de contaminação por bactérias, no paciente submetido à broncoscopia, após o reprocessamento do broncoscópio"
}

\author{
NANCY SPEKLA GRANDE ${ }^{1}$, ROMILDA APARECIDA NAKAYAMA ${ }^{2}$, \\ ANTONIA MARIA DE OlIVEIRA MACHADO ${ }^{3}$, FÁBIO AKIO YAMAGUTI $^{4}$, CESAR UEHARA $^{5}$
}

Introdução: A broncoscopia é um procedimento diagnóstico e terapêutico realizado com a introdução nas vias aéreas de um tubo flexível que permite visualizar até as menores delas. Para evitar ou reduzir o risco de infecções, o broncoscópio deve ser adequadamente desinfetado com, pelo menos, desinfecção de alto nível. Objetivo: Verificar se há risco de contaminação bacteriana para os pacientes submetidos à broncoscopia do Hospital São Paulo da Unifesp, Estado de São Paulo.

Métodos: No período de 1997 a 1998, o reprocessamento do broncoscópio incluiu limpeza e enxágüe com água estéril ou potável, seguida de rinsagem com glutaraldeído a $2 \%$ por 20 minutos, novo enxágüe com água estéril ou potável e rinsagem com álcool etílico a $70 \%$ e secagem com ar forçado pelo canal de sucção. Foram colhidas amostras de 65 pacientes para exames microbiológicos, instalando-se soro fisiológico estéril pelo canal de sucção do broncoscópio. Resultados: Após o reprocessamento foram encontrados nas amostras Staphylococcus epidermidis, Enterobacter sp, Acinetobacter baumanni, Streptococcus viridans, Staphylococcus aureus, Streptococcus beta hemoliticus A, Staphylococcus coagulase negativa e em cinco amostras houve crescimento de microbactéria na cultura. Conclusões: A desinfecção do broncoscópio com glutaraldeído a $2 \%$ não foi suficiente para garantir a desinfecção do aparelho e a presença de Staphylococcus epidermidis indica que houve contaminação do broncoscópio pela manipulação após a desinfecção.

(J Pneumol 2002;28(5):250-60)

\section{Evaluation of the risk of bacterial contamination in the patient submitted to bronchoscopy, after reprocessing the bronchoscope}

Introduction: Bronchoscopic is a diagnostic and therapeutic procedure performed by introducing a flexible tube in the airways that allows for the visualization even of the smallest airways. In order to prevent or reduce the risk of infection, the bronchoscope must be adequately disinfected with at least high level disinfection. Purpose: Check for the risk of bacterial contamination for patients submitted to bronchoscopy at the Hospital São Paulo of Unifesp University, State of São Paulo. Methods: From 1997 to 1998, bronchoscope reprocessing included cleaning, rinsing with sterile or potable water, followed by rinsing with $2 \%$ glutaraldehyde for 20 minutes, rinsing again with sterile or potable water, and rinsing with $70 \%$ ethyl alcohol, and forced-air drying through the suction channel. Samples of 65 patients were collected for microbiologic tests by instilling sterile saline solution through the suction channel of

the bronchoscope. Results: After reprocessing, the following were found in the samples:

Staphylococcus epidermidis, Enterobacter sp, Acinetobacter baumanni, Streptococcus viridans, Staphylococcus aureus, Streptococcus beta hemoliticus A, Staphylococcus coagulase negative, and mycobacterial growth was found in the culture of five samples. Conclusions: Bronchoscope disinfection with $2 \%$ glutaraldehyde was not sufficient to assured disinfection of the scope and the presence of Staphylococcus epidermidis shows that there was bronchoscope contamination due to handling after disinfection.

* Trabalho realizado na Universidade Federal do Estado de São Paulo (Unifesp/EPM), São Paulo, SP.

1. Enfermeira da Unidade de Pneumologia do HSP. Mestre em Ciências Pneumológicas.

2. Médica Broncoscopista do Hospital São Paulo.

3. Médica Responsável pelo Setor de Microbiologia do Laboratório Central do HSP.
4. Médico Assistente da UTI do HC-FMB-Unesp.

5. Professor Adjunto da Disciplina de Pneumologia.

Endereço para correspondência - Nancy Spekla Grande, Rua Botucatu, 740, $3^{\circ}$ and. - Disciplina de Pneumologia - 04023-062 - São Paulo, SP.

Recebido para publicação em 2/10/01. Aprovado, após revisão, em 17/1/02. 


\section{INTRODUÇÃO}

Antigamente, acreditava-se que a broncoscopia tivesse baixo ou nenhum risco de infecção, mas, com os adventos da SIDA e da tuberculose epidêmica, começou-se a pensar em infecção pelo broncoscópio ${ }^{(1,2)}$. Existe risco potencial de transmissão de infecção durante a broncoscopia e o espectro dessas infecções vai da colonização assintomática à morte ${ }^{(1,3)}$.

Rotineiramente, durante seu uso, os broncoscópios são contaminados por microorganismos. Técnica desapropriada ou falhas durante o reprocessamento do broncoscópio podem causar infecção nosocomial, principalmente em pacientes imunocomprometidos ${ }^{(4)}$.

As vias aéreas superiores são colonizadas por floras bacterianas que vão escasseando até chegar aos brônquios, que, usualmente, são estéreis. Pereira et al. ${ }^{(5)}$ encontraram em um estudo com 66 pacientes, pós-broncoscopia, um com pneumonia, em quem foi isolado microorganismo da flora oral.

Na literatura são relatados casos de aumento da temperatura até oito dias após a broncoscopia ${ }^{(6-14)}$. Esse aumento da temperatura pode estar relacionado à toxina das bactérias, quando há infecção, ou mesmo ao aumento do fator de necrose tumoral no lavado broncoalveolar(15), o que torna difícil a elucidação de infecção via broncoscópio.

Há relatos que sugerem que o broncoscópio pode transmitir infecção via transbrônquica. Os achados foram: piora do infiltrado preexistente ou desenvolvimento de novos infiltrados ${ }^{(16)}$, um caso de pneumonia estreptocócica e septicemia nas 24 horas após a broncoscopia com biópsia endobrônquica(17), aspiração de um abscesso pulmonar ${ }^{(18)}$, lobectomia de um abscesso pulmonar desenvolvido sete dias após biópsia transbrônquica ${ }^{(19)}$, pneumonia bacteriana progressiva em dois pacientes e um abscesso pulmonar por anaeróbios em um paciente com criptococose, após broncoscopias ${ }^{(20)}$. Há vários relatos de tuberculose progredindo dias a semanas após a broncoscopia, culminando com a morte em alguns casos $^{(19-22)}$.

O broncoscópio é um artigo semicrítico, por estar em contato com a mucosa íntegra. Devido a sua estrutura complexa e frágil, que inclui partes constituídas de materiais sensíveis ao calor, o broncoscópio impõe restrições a certos métodos de reprocessamento. Os mais apropriados para esse aparelho (item semicrítico) são a esterilização por óxido de etileno, que leva um tempo de aeração de $24 \mathrm{~h}$ ou a esterilização com soluções quimioesterilizantes (glutaraldeído a 2\%) em imersão por 8 a 10h. Estas técnicas são inviáveis aos serviços de grande movimento, como é o caso do Serviço de Broncoscopia do Hospital São Paulo. Ou, ainda, pode-se realizar a desinfecção de alto nível com glutaraldeído a $2 \%$ por 20 minutos, sendo este tempo o mínimo recomendado ${ }^{(23,24)}$.
Siglas e abreviaturas utilizadas neste trabalho

SIDA - Síndrome da imunodeficiência adquirida

CDC - Centers for Disease Control

NCCLS - National Comitee for Clinical Laboratory Standard

As técnicas usualmente recomendadas para o reprocessamento do broncoscópio são a desinfecção com solução de glutaraldeído a $2 \%$ ou esterilização com óxido de etileno ${ }^{(25)}$. Segundo Dawson et al. ${ }^{(26)}$ e Leers ${ }^{(27)}$, o instrumento deve ser imerso na solução de glutaraldeído por 20 a 30 minutos; ou exposto a óxido de etileno durante 90 minutos, seguido de aeração por 10 horas. A desinfecção química com glutaraldeído a $2 \%$, na temperatura de $25^{\circ} \mathrm{C}$ com tempo de exposição por 45 minutos, é $100 \%$ efetiva para destruir $M$. tuberculosis e cai para 99,8\%, se o tempo de exposição baixa para 10 minutos.

O Centers for Disease Control (CDC), assim como o Ministério da Saúde e a Secretaria de Saúde do Estado de São Paulo, recomendam como procedimento mínimo uma desinfecção de alto nível para esse aparelho(28-35).

Com vistas no que foi exposto até agora, resolvemos realizar este trabalho, objetivando verificar se há risco de contaminação por bactérias para o paciente que se submete a broncoscopia, após a desinfecção do broncoscópio na Unidade de Broncoscopia do Hospital São Paulo, no período de 1997 a 1998.

\section{CASUÍSTICA E MÉTODO}

Este trabalho baseou-se na experiência diária do serviço e em relatos de outro serviço de broncoscopia, em que se observaram exames falso-positivos de BK no lavado brônquico, devido à contaminação do broncoscópio. Apesar de não termos relatos no nosso serviço, optamos por realizar controle laboratorial no sentido de verificar se o procedimento de limpeza e desinfecção do aparelho estava sendo eficiente.

As amostras foram coletadas entre 1997 e 1998, de exames broncoscópicos de 65 pacientes que vinham encaminhados para diagnóstico de sua doença através do material colhido de rotina, durante o exame. São colhidos de rotina, durante a broncoscopia, lavado brônquico ou broncoalveolar e biópsia brônquica ou transbrônquica. O aparelho utilizado foi o broncoscópio Olympus $P 20$.

As patologias e/ou doenças de base constituíam-se de hipóteses diagnósticas; a maioria dos pacientes veio para elucidação diagnóstica, ou seja, havia suspeita de câncer de pulmão, tuberculose, infecções oportunistas em pacientes soropositivos para HIV, infecções bacterianas, micoses pulmonares, fibrose pulmonar; não estavam em uso de antibioticoterapia os pacientes. 
Vinte e três pacientes $(35,4 \%)$ eram do sexo feminino e $42(64,6 \%)$ do masculino. A idade variava de dois a 83 anos, sendo a média de 49 anos.

No Hospital São Paulo, as broncoscopias são realizadas rotineiramente, em um local reservado para este procedimento, em pacientes internados e ambulatoriais. Entre um paciente e outro, o aparelho é submetido a uma desinfecção de alto nível com glutaraldeído a $2 \%$ por 20 minutos realizado por profissional não médico treinado, ou pelo médico broncoscopista, e que segue o seguinte protocolo ${ }^{(36-40)}$.

- A limpeza deve ser realizada após cada procedimento, com o uso de água corrente e detergente neutro, devendo-se escovar e lavar todos os canais e conexões do aparelho, deixando-o previamente submerso em detergente enzimático (Endozime) por um período de três a cinco minutos.

- Secar o aparelho antes de ser colocado no glutaraldeído a $2 \%$, para evitar a rediluição do desinfetante.

- Altos níveis de desinfecção devem ser obtidos com o uso do glutaraldeído a $2 \%$. Após o uso do aparelho em cada paciente, e antes do primeiro exame do dia, este deve ser submetido a uma submersão em glutaraldeído a $2 \%$ por 20 minutos.

- Após desinfecção, o aparelho e seus canais devem ser enxaguados com água estéril, ou com água potável, seguida com rinsagem com álcool a 70\%.

- O canal deve ser seco através de fluxo de oxigênio.

- A estocagem do aparelho deve ser feita em ambiente seco, na posição vertical e com ventilação adequada.

Durante a introdução do aparelho, podemos levar material da orofaringe, inclusive as bactérias residentes, para a árvore traqueobrônquica. Estas bactérias, do ponto de vista de infecção, têm pouca importância. Apenas podem confundir quando se deseja identificar o agente etiológico da infecção bacteriana utilizando-se apenas cultura qualitativa.

Durante a realização da broncoscopia em pacientes infectados há acúmulo de secreção e de agentes patogênicos no canal de aspiração e, se não realizarmos limpeza e desinfecção adequadas, podemos levar infecção a outros pacientes.

Como pré-medicação, foi rotineiramente usado $0,5 \mathrm{mg}$ de sulfato de atropina e $50 \mathrm{mg}$ de meperidina ou $5 \mathrm{mg}$ de diazepam ou midazolam por via endovenosa, minutos antes do início do exame.

Com o paciente em decúbito dorsal foi aplicada anestesia tópica na orofaringe com lidocaína spray a $10 \%$, em três séries de três borrifadas, que corresponderam a cerca de 90mg de lidocaína base, no total. Cada série de sprays foi feita a intervalo de um minuto, aproximadamente.
Para a introdução do aparelho foi utilizada a via nasal, sendo que as narinas haviam sido untadas previamente com lidocaína geléia a $2 \%$, com a finalidade anestésica e lubrificante; ou a via endotraqueal, quando o paciente estava intubado.

Através do canal de aspiração do broncofibroscópio locado na orofaringe, foi instilada lidocaína a $2 \%$, em porções de $1 \mathrm{ml}$, nas paredes das laringe, até que se tivesse obtido adequada inibição do reflexo da tosse. A dose utilizada, em geral, foi de 5 a $8 \mathrm{ml}^{(41,42)}$.

Ultrapassada a laringe, o aparelho era deslizado para as vias aéreas inferiores, alternando avanço do aparelho e instilação de $1 \mathrm{ml}$ de lidocaína líquida a $2 \%$, evitando exceder a dose total de $400 \mathrm{mg}$.

Com o broncoscópio posicionado, para a coleta do lavado broncoalveolar, eram instilados lotes de $20 \mathrm{ml}$ de soro fisiológico estéril e, em seguida, aspirado com sucção controlada, para evitar o colapso das vias aéreas, direto a um tubo de Lukens estéril. O volume total do soro fisiológico administrado era de 100 a $180 \mathrm{ml}$.

Coletamos do canal do broncoscópio material para pesquisa de $\mathrm{BK}$, bacterioscopia, cultura bacteriana e de micobactéria em três fases:

- antes da realização da broncoscopia, para verificar se o broncoscópio estava contaminado ou não antes de entrar em contato com o paciente. Este era enumerado frasco 1.

- imediatamente após a realização da broncoscopia, para saber qual a flora retida no broncoscópio após o término do exame. Este era enumerado frasco 3.

- após a desinfecção de alto nível, para avaliar se esta foi realmente eficaz. Este era enumerado frasco 4.

Para enriquecer os resultados aproveitamos os resultados do lavado brônquico ou broncoalveolar do paciente, que fazia parte do procedimento habitual do exame. Este era enumerado frasco 2.

Logo em seguida, os tubos devidamente identificados eram encaminhados para o Laboratório Central do Hospital São Paulo, Setor de Microbiologia, onde eram manipulados em fluxo laminar, preparadas lâminas para detecção do bacilo de Koch e feita a coloração para gram, preparadas placas de Teague, ágar sangue e ágar chocolate e encaminhada parte da amostra para cultura de micobactéria. Tudo segundo normas internacionais da NCCLS (National Committee for Clinical Laboratory Standard $)^{(43)}$.

Foram tabuladas todas as amostras e atribuídos sinais $(+)$ quando houve crescimento nesse frasco ou (-) quando não houve crescimento; $e$ feitas tabelas para cada pesquisa gram, BK, aeróbios e micobactérias.

Para análise dos resultados aplicamos o teste $\mathrm{G}$ de Cochran ${ }^{(44)}$ para estudar a concomitância da presença/ 
ausência de cada microorganismo, nos quatro tubos estudados.

Fixamos em 0,05 ou $5 \%(\alpha \leq 0,05)$ o nível de rejeição da hipótese de nulidade, assinalando-se com um asterisco os valores significativos.

\section{RESULTADOS}

Os resultados de BAAR foram negativos em todos os frascos das amostras; portanto, o $\mathrm{G}$ de Cochran mostrou-se não-significativo. O BAAR foi o exame para detecção de BK de mais rápido resultado (Tabela 1 ).

$\mathrm{Na}$ cultura de micobactérias houve crescimentos em vários frascos. Somente Mycobacterium tuberculosis cresceu na cultura.

\begin{tabular}{|c|c|c|c|c|c|c|c|c|c|}
\hline \multirow{3}{*}{ Amostra } & \multicolumn{5}{|c|}{$\begin{array}{c}\text { TABELA } 1 \\
\text { BAAR }\end{array}$} & \multirow{2}{*}{\multicolumn{4}{|c|}{ Frascos }} \\
\hline & \multicolumn{4}{|c|}{ Frascos } & \multirow[t]{2}{*}{ Amostra } & & & & \\
\hline & 1 & 2 & 3 & 4 & & 1 & 2 & 3 & 4 \\
\hline 1 & - & - & - & - & 35 & - & - & - & - \\
\hline 2 & - & - & - & - & 36 & - & - & - & - \\
\hline 3 & - & - & - & - & 37 & - & - & - & - \\
\hline 4 & - & - & - & - & 38 & - & - & - & - \\
\hline 5 & - & - & - & - & 39 & - & - & - & - \\
\hline 6 & - & - & - & - & 40 & - & - & - & - \\
\hline 7 & - & - & - & - & 41 & - & - & - & - \\
\hline 8 & - & - & - & - & 42 & - & - & - & - \\
\hline 9 & - & - & - & - & 43 & - & - & - & - \\
\hline 10 & - & - & - & - & 44 & - & - & - & - \\
\hline 11 & - & - & - & - & 45 & - & - & - & - \\
\hline 12 & - & - & - & - & 46 & - & - & - & - \\
\hline 13 & - & - & - & - & 47 & - & - & - & - \\
\hline 14 & - & - & - & - & 48 & - & - & - & - \\
\hline 15 & - & - & - & - & 49 & - & - & - & - \\
\hline 16 & - & - & - & - & 50 & - & - & - & - \\
\hline 17 & - & - & - & - & 51 & - & - & - & - \\
\hline 18 & - & - & - & - & 52 & - & - & - & - \\
\hline 19 & - & - & - & - & 53 & - & - & - & - \\
\hline 20 & - & - & - & - & 54 & - & - & - & - \\
\hline 21 & - & - & - & - & 55 & - & - & - & - \\
\hline 22 & - & - & - & - & 56 & - & - & - & - \\
\hline 23 & - & - & - & - & 57 & - & - & - & - \\
\hline 24 & - & - & - & - & 58 & - & - & - & - \\
\hline 25 & - & - & - & - & 59 & - & - & - & - \\
\hline 26 & - & - & - & - & 60 & - & - & - & - \\
\hline 27 & - & - & - & - & 61 & - & - & - & - \\
\hline 28 & - & - & - & - & 62 & - & - & - & - \\
\hline 29 & - & - & - & - & 63 & - & - & - & - \\
\hline 30 & - & - & - & - & 64 & - & - & - & - \\
\hline 31 & - & - & - & - & 65 & - & & - & - \\
\hline $\begin{array}{l}32 \\
33\end{array}$ & - & - & - & - & Total & 0 & 0 & 0 & 0 \\
\hline 34 & - & - & - & - & $\%$ & 0 & 0 & 0 & $\overline{0}$ \\
\hline
\end{tabular}

Os resultados de BAAR dispensam análise
O cálculo do G de Cochran mostrou-se significante no frasco 1, por este apresentar menor índice de contaminação que os demais frascos (4,6\%) (Tabela 2).

Houve também crescimento em $15,4 \%$ dos frascos 2 , $10,8 \%$ dos frascos 3 e $7,7 \%$ dos frascos 4 .

Houve crescimento de Mycobacterium tuberculosis nos frascos 2 e 3 em três amostras. Houve crescimento nos frascos 2, 3 e 4 em duas amostras. Em duas amostras houve crescimento em todos os frascos. Em uma amostra houve crescimento no frasco 2 ; e em uma amostra houve crescimento nos frascos 1,2 e 4 .

Para os resultados de gram mostraram-se significantes os frascos 1 e frascos 4 (Tabela 3).

TABELA 2

Micobactérias

\begin{tabular}{|c|c|c|c|c|c|c|c|c|c|}
\hline \multirow[t]{2}{*}{ Amostra } & \multicolumn{4}{|c|}{ Frascos } & \multirow[t]{2}{*}{ Amostra } & \multicolumn{4}{|c|}{ Frascos } \\
\hline & 1 & 2 & 3 & 4 & & 1 & 2 & 3 & 4 \\
\hline 1 & - & - & - & - & 35 & - & - & - & - \\
\hline 2 & - & - & - & - & 36 & - & - & - & - \\
\hline 3 & - & - & - & - & 37 & - & - & - & - \\
\hline 4 & - & - & - & - & 38 & - & - & - & - \\
\hline 5 & - & - & - & - & 39 & - & - & - & - \\
\hline 6 & - & + & + & - & 40 & - & - & - & - \\
\hline 7 & - & + & + & + & 41 & - & - & - & - \\
\hline 8 & + & + & - & + & 42 & - & + & + & - \\
\hline 9 & - & - & - & - & 43 & - & + & + & - \\
\hline 10 & - & - & - & - & 44 & - & - & - & - \\
\hline 11 & - & - & - & - & 45 & - & - & - & - \\
\hline 12 & - & - & - & - & 46 & - & - & - & - \\
\hline 13 & - & - & - & - & 47 & - & - & - & - \\
\hline 14 & - & - & - & - & 48 & - & - & - & - \\
\hline 15 & - & - & - & - & 49 & - & - & - & - \\
\hline 16 & - & - & - & - & 50 & - & - & - & - \\
\hline 17 & - & - & - & - & 51 & - & + & + & + \\
\hline 18 & - & - & - & - & 52 & - & - & - & - \\
\hline 19 & - & - & - & - & 53 & + & + & + & + \\
\hline 20 & - & - & - & - & 54 & - & - & - & - \\
\hline 21 & - & - & - & - & 55 & - & - & - & - \\
\hline 22 & - & - & - & - & 56 & - & - & - & - \\
\hline 23 & - & - & - & - & 57 & - & - & - & - \\
\hline 24 & - & - & - & - & 58 & - & - & - & - \\
\hline 25 & - & - & - & - & 59 & - & - & - & - \\
\hline 26 & - & - & - & - & 60 & - & - & - & - \\
\hline 27 & - & - & - & - & 61 & - & - & - & - \\
\hline 28 & - & - & - & - & 62 & + & + & + & + \\
\hline 29 & - & - & - & - & 63 & - & - & - & - \\
\hline 30 & - & - & - & - & 64 & - & + & - & - \\
\hline 31 & - & + & - & - & 65 & - & - & - & - \\
\hline $\begin{array}{l}32 \\
33\end{array}$ & - & - & - & - & Total & 3 & 10 & 7 & 5 \\
\hline 34 & - & - & - & - & $\%$ & 46 & 154 & 108 & 7,7 \\
\hline
\end{tabular}

Teste G de Cochran

G calculado: $11,89^{*}$ - G crítico: 7,82 
Não houve crescimento nos frascos 1 , como era esperado. Mas houve um caso $(1,5 \%)$ de contaminação no frasco 4 , isto é, após a desinfecção de alto nível.

Foram encontrados $35,4 \%$ de casos positivos no lavado broncoalveolar do paciente, que se mantiveram positivos em $20 \%$ antes da desinfecção de alto nível (frasco 3). A grande maioria desses era gram+.

$\mathrm{Na}$ cultura de aeróbios, o cálculo do $\mathrm{G}$ de Cochran mostrou-se significativo nos frascos $1(9,2 \%)$ e $4(16,9 \%)$, por estes apresentarem o menor número de amostras positivas (Tabela 4).

Houve crescimento no frasco 1 de seis amostras: uma com Streptococcus viridans e cinco com Staphylococ- cus coagulase negativa. O broncoscópio chegou contaminado em 9,2\% das amostras.

Houve crescimento no frasco 2 de 62 amostras. A flora encontrada nos pacientes foi a seguinte:

$\begin{array}{lc}\text { Streptococcus viridans } & 35(53,8 \%) \\ \text { Staphylococcus aureus } & 13(20 \%) \\ \text { Staphylococcus coagulase negativa } & 9(13,8 \%) \\ \text { Pseudomonas aeruginosa } & 8(12,3 \%) \\ \text { Klebsiella pneumoniae } & 8(12,3 \%) \\ \text { Enterobacter sp } & 4(6,2 \%) \\ \text { Enterococcus sp } & 3(4,6 \%) \\ \text { Escherichia coli } & 2(3,1 \%) \\ \text { Streptococcus betahemolitic A } & 2(3,1 \%)\end{array}$

\begin{tabular}{|c|c|c|c|c|c|c|c|c|c|}
\hline \multirow[t]{2}{*}{ Amostra } & \multicolumn{4}{|c|}{ Frascos } & \multirow[t]{2}{*}{ Amostra } & \multicolumn{4}{|c|}{ Frascos } \\
\hline & 1 & 2 & 3 & 4 & & 1 & 2 & 3 & 4 \\
\hline 1 & - & + & - & - & 35 & - & - & - & - \\
\hline 2 & - & + & - & - & 36 & - & - & - & - \\
\hline 3 & - & + & + & - & 37 & - & - & - & - \\
\hline 4 & - & + & - & - & 38 & - & - & - & - \\
\hline 5 & - & - & - & - & 39 & - & + & - & - \\
\hline 6 & - & - & - & - & 40 & - & - & - & - \\
\hline 7 & - & + & - & - & 41 & - & - & - & - \\
\hline 8 & - & + & + & + & 42 & - & - & - & - \\
\hline 9 & - & - & - & - & 43 & - & - & - & - \\
\hline 10 & - & - & - & - & 44 & - & + & - & - \\
\hline 11 & - & - & - & - & 45 & - & - & - & - \\
\hline 12 & - & + & + & - & 46 & - & + & + & - \\
\hline 13 & - & + & - & - & 47 & - & - & - & - \\
\hline 14 & - & - & - & - & 48 & - & + & - & - \\
\hline 15 & - & - & - & - & 49 & - & + & + & - \\
\hline 16 & - & - & - & - & 50 & - & + & + & - \\
\hline 17 & - & - & - & - & 51 & - & - & - & - \\
\hline 18 & - & - & - & - & 52 & - & - & - & - \\
\hline 19 & - & - & - & - & 53 & - & - & + & - \\
\hline 20 & - & - & - & - & 54 & - & - & - & - \\
\hline 21 & - & - & - & - & 55 & - & - & - & - \\
\hline 22 & - & + & + & - & 56 & - & + & + & - \\
\hline 23 & - & - & - & - & 57 & - & + & - & - \\
\hline 24 & - & + & - & - & 58 & - & - & - & - \\
\hline 25 & - & - & - & - & 59 & - & - & + & - \\
\hline 26 & - & - & - & - & 60 & - & - & - & - \\
\hline 27 & - & + & - & - & 61 & - & + & - & - \\
\hline 28 & - & - & - & - & 62 & - & - & + & - \\
\hline 29 & - & - & - & - & 63 & - & - & + & - \\
\hline 30 & - & - & - & - & 64 & - & + & - & - \\
\hline 31 & - & + & + & - & 65 & - & - & - & - \\
\hline 32 & - & - & - & - & Total & 0 & 23 & 13 & 1 \\
\hline 33 & - & - & - & - & & & & & \\
\hline 34 & - & - & - & - & $\%$ & 0 & 35,4 & 20 & 15 \\
\hline
\end{tabular}

Teste G de Cochran

G calculado: $48,1^{*}$ - G crítico: 7,82
TABELA 4

Cultura de aeróbios

GRAM

TABELA 3

Amostra

\begin{tabular}{cccc}
\multicolumn{4}{c}{ Frascos } \\
\hline 1 & 2 & 3 & 4
\end{tabular}

\section{1}

1
2

2
3

3
5

5
6

7
8

8
9

10
11

12

13

14
15

15
16

17
18

18
19

$$
20
$$

21$$
\begin{aligned}
& 22 \\
& 23
\end{aligned}
$$$$
24
$$$$
25
$$$$
26
$$$$
\begin{aligned}
& 27 \\
& 28
\end{aligned}
$$$$
\begin{aligned}
& 28 \\
& 29
\end{aligned}
$$$$
30
$$$$
31
$$$$
32
$$$$
\begin{aligned}
& 33 \\
& 34
\end{aligned}
$$

\section{$-\quad+\quad+\quad-$}

$\begin{array}{cccc}- & + & + & - \\ - & + & + & - \\ - & + & + & +\end{array}$

$-+++$

$-++-$$$
-++\quad-
$$$$
\begin{array}{llll}
- & + & + & - \\
- & + & + & -
\end{array}
$$$$
\begin{array}{llll}
+ & - & + & - \\
- & + & + & +
\end{array}
$$$$
\begin{array}{llll}
- & + & + & + \\
- & + & + & -
\end{array}
$$$$
\begin{array}{llll}
- & + & + & - \\
- & + & + & -
\end{array}
$$$$
\begin{array}{llll}
- & + & + & - \\
- & + & + & -
\end{array}
$$$$
-++
$$$$
\begin{array}{llll}
- & + & + & - \\
- & + & + & +
\end{array}
$$$$
\begin{array}{llll}
- & + & + & + \\
- & + & + & -
\end{array}
$$$$
\begin{array}{llll}
- & + & + & - \\
- & + & + & +
\end{array}
$$$$
\begin{array}{llll}
- & + & + & + \\
- & + & + & -
\end{array}
$$$$
\begin{array}{llll}
- & + & + & - \\
- & + & + & -
\end{array}
$$$$
\begin{array}{llll}
- & + & + & - \\
- & + & + & -
\end{array}
$$$$
\begin{array}{llll}
- & + & + & - \\
- & - & + & -
\end{array}
$$$$
-\quad-+-
$$$$
-++-
$$$$
-++
$$$$
\begin{array}{llll}
- & + & + & - \\
- & + & + & - \\
- & + & + & +
\end{array}
$$$$
\begin{array}{llll}
- & + & + & + \\
- & + & + & -
\end{array}
$$$$
\begin{array}{llll}
- & + & + & - \\
- & + & + & -
\end{array}
$$$$
\begin{array}{llll}
- & + & + & - \\
- & + & + & -
\end{array}
$$$$
\begin{array}{llll}
- & + & + & - \\
- & +
\end{array}
$$$$
-+++
$$$$
\begin{array}{ccccc}
\text { Amostra } & \multicolumn{5}{c}{\text { Frascos }} \\
\cline { 2 - 5 } & \mathbf{1} & \mathbf{2} & \mathbf{3} & \mathbf{4} \\
\hline 35 & - & + & - & + \\
36 & + & + & + & - \\
37 & - & + & + & - \\
38 & - & + & + & - \\
39 & - & + & + & - \\
40 & - & + & + & - \\
41 & - & + & + & - \\
42 & - & + & + & - \\
43 & - & + & + & - \\
44 & - & + & + & - \\
45 & - & + & + & + \\
46 & + & + & + & - \\
47 & - & + & + & - \\
48 & - & + & + & - \\
49 & - & + & + & - \\
50 & - & + & + & - \\
51 & - & + & + & - \\
52 & - & + & + & - \\
53 & - & + & + & - \\
54 & - & + & + & - \\
55 & + & + & + & - \\
56 & - & + & + & + \\
57 & - & + & + & - \\
58 & - & + & + & + \\
59 & + & + & + & - \\
60 & - & + & + & - \\
61 & - & + & + & - \\
62 & + & + & + & + \\
63 & - & + & + & + \\
64 & - & + & + & + \\
65 & - & + & + & + \\
\hline \text { Total } & 6 & 62 & 63 & 11 \\
\hline \% & 92 & 95,4 & 969 & 169 \\
& & & &
\end{array}
$$

Teste G de Cochran

G calculado: $146,45^{*}$ - G crítico: 7,82$$
\begin{array}{llll}
- & + & + & - \\
+ & - & + & -
\end{array}
$$$$
\begin{array}{llll}
- & + & + & - \\
- & + & + & -
\end{array}
$$$$
\begin{array}{llll}
- & + & + & - \\
- & + & + & -
\end{array}
$$$$
-+++
$$ 


Acinetobacter baumanni
Corinebacterium sp
Citrobacter $s p$
Proteus mirabilis

Houve crescimento no frasco 3 de 63 amostras. Isto é: após o exame, o broncoscópio reteve microorganismos em $96,9 \%$ das amostras. Foram achados:

Pseudomonas aeruginosa
Streptococcus viridans
Klebsiella pneumoniae
Enterobacter sp
Staphylococcus aureus
Acinetobacter baumanni
Enterococcus sp
Staphylococcus coagulase negativa
Staphylococcus epidermidis
Streptococcus pneumoniae
Escherichia coli
Providencia rett-geri
Corinebacterium sp
Stenotrophomonas (xant. Maltophilia)
Streptococcus betahemolit. A

$9(13,8 \%)$

$8(12,3 \%)$

$8(12,3 \%)$

$5(7,7 \%)$

$4 \quad(6,2 \%)$

$3(4,6 \%)$

$3(4,6 \%)$

$2(3,1 \%)$

$2(3,1 \%)$

$2(3,1 \%)$

$1(1,54 \%)$

$1(1,54 \%)$

$1(1,54 \%)$

Após a desinfecção, o broncoscópio manteve-se contaminado em 11 amostras (16,9\%), nas quais foram achados:

Staphylococcus coagulase negativa
Staphylococcus epidermidis
Enterobacter sp
Acinetobacter baumanni
Streptococcus viridans
Staphylococcus aureus
Pseudomonas aeruginosa
Streptococcus betahemolit. A

Quando comparamos a bacterioscopia com a bacteriologia, no frasco 1, verificamos que, apesar de a bacterioscopia ter se apresentado negativa em todas as amostras, houve crescimento de microorganismos na cultura de seis amostras (casos 8, 36, 46, 55, 59 e 62).

Houve três casos de gram positivo e cultura negativa no frasco 2 (casos 8, 22, 31). Em 42 amostras, gram foi negativo nesse frasco, mas teve a cultura positiva.

Houve 13 casos de gram positivo (casos $3,8,12,22$, $31,46,49,50,53,56,59,62$ e 63) e dois de cultura negativa, no frasco 3 (casos 33 e 34).

No frasco 4 houve um caso de gram positivo e cultura negativa (caso 8), que pode indicar que a bactéria não resistiu até ser semeada. No frasco 4 foram encontrados três casos de Staphylococcus epidermidis.

\section{DISCUSSÃO}

Teoricamente, o broncoscópio apresenta grande risco de causar complicações porque o instrumento atravessa a orofaringe e carrega microorganismos para as vias aéreas inferiores e para o parênquima pulmonar.

Nos Estados Unidos da América, as infecções de trato respiratório baixo somam aproximadamente 20\% das infecções hospitalares, com 200.000 casos por ano. Mais da metade dessas infecções são polimicrobianas por organismos gram-, como Pseudomonas aeruginosa, Klebsiella sp, Enterobacter sp, Escherichia coli, Serratia sp, Proteus sp.; 70\% são aeróbios gram+e a maioria é Staphylococcus coagulase positiva ${ }^{(45)}$.

O lavado broncoalveolar tem benefício confirmado no diagnóstico de patógenos pulmonares oportunistas; entretanto, a contaminação do lavado pela flora da orofaringe pode falsear os resultados das culturas do material coletado por essa técnica.

O reprocessamento do broncoscópio deve abranger as seguintes etapas, segundo Rutala ${ }^{(46)}$ :

- A limpeza mecânica com água e detergente neutro e enzimático tem como objetivo a retirada da matéria orgânica da superfície externa e dos canais do broncoscópio. $\mathrm{O}$ uso de detergente diminui a tensão superficial da matéria orgânica, permitindo a suspensão de suas partículas. As enzimas proteolíticas no detergente enzimático quebram as proteínas da matéria orgânica, removendo-a $e$ facilitando a limpeza.

- Enxágüe e drenagem dos canais, permitindo a retirada do detergente com a sujeira e escoamento da água $e$ secagem do canal, impedindo a alteração do $\mathrm{pH}$ e a rediluição do desinfetante.

- Desinfecção em alto nível com glutaraldeído a 2\% por 20 minutos, para eliminar microorganismos como o Mycobacterium tuberculosis. O glutaraldeído é um desinfetante ou esterilizante químico, dependendo do tempo em contato com a superfície do objeto a ser reprocessado. Sua ação depende do período de validade (14 ou 28 dias), das condições de uso (temperatura, por exemplo), rediluição e contato com a matéria orgânica ${ }^{(24)}$. Tem amplo espectro de atuação, atividade mesmo em presença de matéria orgânica, embora esta a lentifique.

- Finda a desinfecção, novo enxágüe com água estéril ou potável seguida, neste caso, de rinsagem com álcool a $70 \%$. Objetiva a retirada de desinfetante.

- Secagem dos canais com ar comprimido e estocagem em local apropriado para não haver contaminação ou dano ao aparelho.

Falha em qualquer dessas etapas ou mesmo uso de produtos contaminados podem levar à não eficácia da desinfecção e, conseqüentemente, à contaminação do broncoscópio. 
Alguns fatores podem interferir na qualidade do reprocessamento do broncoscópio. São elas:

- A configuração do aparelho e sua composição.

- O uso de água ou soluções contaminadas.

- Presença de matéria orgânica.

- Qualidade da limpeza.

- Concentração e tempo de exposição ao desinfetante.

- Temperatura e pH durante o processo de desinfecção.

- O tipo e o nível de contaminação.

- Resistência microbiana ao desinfetante.

Estudos controlados têm avaliado a eficácia de diferentes desinfetantes para uso no broncoscópio. Dos três desinfetantes testados, glutaraldeído a $2 \%$, álcool etílico e clorexidina, o mais efetivo foi a clorexidina seguido pelo glutaraldeído a $2 \%$ e o álcool etílico em terceiro lugar.

Segundo Penco e Martin ${ }^{(47)}$, a probabilidade de haver transmissão de infecção por artigos esterilizados parece pequena, mas quando são utilizadas soluções químicas germicidas em artigos que não podem ser submetidos a métodos físicos, essa possibilidade passa a existir. Já para Rutala ${ }^{(46)}$, esterilização é um conceito absoluto e não relativo.

A primeira indicação de seu potencial de ação veio de um estudo de Pepper e Lieberman ${ }^{(48)}$, em 1962, de dialdeídos saturados.

Em 1963, Stonehill et al. ${ }^{(49)}$ defendiam que a solução alcalina de glutaraldeído era rapidamente esporicida e no fim daquele ano, o glutaraldeído já começava a ser comercializado como quimioesterilizante seguro e com tempo de contato mais aceitável na rotina dos hospitais.

Spaulding ${ }^{(50)}$, em 1968, classificou o glutaraldeído como um germicida de alto nível, capaz de produzir esterilização se o tempo de exposição for suficientemente longo. Seu poder de destruir esporos é considerado excelente quando comparado com outros germicidas.

Stonehill et al. ${ }^{(49)}$ e Borick et al. ${ }^{(51)}$ encontraram o tempo de três horas para o glutaraldeído a $2 \%$ destruir esporos de Bacillus sp e Clostridium sp. Rubbo et al.(52) relataram que 99,99\% de esporos de Bacillus anthracis e Clostridium tetani foram eliminados com o tempo de 15 e 30 minutos, respectivamente. Muitos fabricantes, no entanto, têm recomendado de 10 a 12 horas para a esterilização com o glutaraldeído, no caso de haver fatores adversos, como esporos resistentes ou rediluição da solução germicida ou, ainda, inativação desta por matéria orgânica ${ }^{(53)}$.

Na pesquisa de BAAR, deste estudo, não houve resultados positivos. Nos frascos 1 , que representam a condição de desinfecção do broncoscópio antes do exame e nos frascos 4, que representam a eficácia ou não da desinfecção do broncoscópio, como era esperado, não foi detectado o bacilo de Koch.
Foram negativos também os resultados dos frascos 2 , que representam o lavado broncoalveolar do paciente, $e$ dos frascos 3, que indicam se o broncoscópio reteve algum microorganismo antes de ser encaminhado para a desinfecção de alto nível.

Neste trabalho, em cultura de micobactérias, o cálculo do $\mathrm{G}$ de Cochran mostrou-se significante nos frascos 1 , por estes apresentarem o menor índice de crescimento que os demais frascos $(4,6 \%)$. No entanto, por estes frascos representarem o broncoscópio antes do exame, deveriam apresentar culturas negativas, para não haver risco de contaminação do paciente.

Dentre as 65 amostras de lavado broncoalveolar dos pacientes, houve crescimento para micobactéria em 10 amostras (15,4\%).

Houve crescimento em $10,8 \%$ dos frascos 3 , após o término do exame; este número caiu para $7,7 \%$ após a desinfecção de alto nível, por 20 minutos.

Houve três casos em que o aparelho ia desinfetado para o paciente, cujo lavado broncoalveolar apresentou crescimento, ou seja o paciente era portador de tuberculose; o aparelho após o exame retinha o microorganismo, mas a desinfecção mostrou-se eficiente. Dois casos, no entanto, embora o broncoscópio fosse desinfetado para o paciente, também apresentou crescimento no lavado; o broncoscópio retinha o microorganismo, a desinfecção não foi eficiente e houve crescimento no frasco 4.

Em dois casos houve crescimento em todos os frascos, representando ineficiência do processo de desinfecção tanto antes do exame, como ao final da desinfecção de alto nível.

Em duas amostras, somente no frasco 2 houve crescimento, mostrando que o broncoscópio não reteve microorganismos ao final do exame e antes de ir para a desinfecção. Provavelmente, o paciente era paucibacilar.

Em uma amostra, não houve crescimento somente no frasco 3 (após o término do exame), indicando que o broncoscópio não apresentava microorganismos antes de ir para a desinfecção. Curiosamente, no frasco 4 (após a desinfecção de alto nível) dessa mesma amostra, houve crescimento. Talvez houvesse maior diluição da amostra 3 , reduzindo proporcionalmente a quantidade de bactérias, resultando na cultura negativa.

Esses achados também foram observados por Cortese e Prakash ${ }^{(45)}$, quando três pacientes investigados durante uma semana tiveram cultura positiva para o mesmo Mycobacterium intracellulare. O acompanhamento desses pacientes permitiu verificar que somente o primeiro era o verdadeiro excretor da micobactéria; os isolados dos outros pacientes eram falso-positivos, causados pela inadequada desinfecção do broncoscópio. Os mesmos autores referem que a broncoscopia flexível, incluindo os re- 
sultados de cultura de material obtido por escovados, lavagens e biópsia em pacientes com tuberculose, terá um sucesso de 58 a $96 \%$ em isolar micobactéria.

Baughman et al. ${ }^{(54)}$ relataram incidência de $68 \%$ de BAAR positivo em material colhido na broncoscopia.

Davis et al. ${ }^{(55)}$ relatam culturas negativas para $M$. gordonae e M. tuberculosis após 15 minutos de desinfecção em glutaraldeído a $2 \%$ ou iodóforo.

Segundo Hanson et al. ${ }^{(23)}$, a limpeza mecânica minuciosa é suficiente para remover virtualmente todos os agentes infecciosos presentes no aparelho e a não realização eficiente desta permite a secagem de matéria orgânica, impossibilitando a penetração do desinfetante, mesmo durante imersão prolongada. Estudo de Davis et al. ${ }^{(55)}$ indicou que limpeza mecânica seguida de 15 minutos de imersão no glutaldeído a $2 \%$ e enxágüe em água estéril foram suficientes para eliminar efetivamente $M$. tuberculosis e M. gordonae do broncoscópio contaminado artificialmente.

Carson et al. ${ }^{(56)}$ mostraram que cepas TM de Mycobacterium chelonei sobreviveram à exposição por 60 minutos em glutaraldeído a 2\%. Enquanto cepas ATCC não sobreviveram à exposição por dois minutos ao mesmo desinfetante. Isso demonstra a vulnerabilidade de certas cepas de microorganismo, enquanto outras são resistentes ao mesmo desinfetante.

A desinfecção química com glutaraldeído a $2 \%$, à temperatura de $25^{\circ} \mathrm{C}$, com tempo de imersão de 45 minutos, permite eficácia de $100 \%$ na eliminação do $M$. tuberculosis. Essa eficácia cai para 99,8\%, se o tempo de exposição baixar para 10 minutos. A exposição por 10 minutos é eficiente para matar organismos vegetativos e HIV(55). Mas neste trabalho avaliamos o método de reprocessamento que é utilizado no dia-a-dia do serviço de broncoscopia, isto é, a desinfecção de alto nível com glutaraldeído a $2 \%$ por 20 minutos, que, como já foi mencionado, é o mínimo recomendado por vários órgãos. Em 65 amostras de lavado broncoalveolar do paciente, houve crescimento de Mycobacterium tuberculosis em 10 amostras. Após o término do exame o broncoscópio reteve microorganismos em sete amostras; destes, a desinfecção foi eficiente em apenas três amostras. A confrontação destes achados com a literatura mostra que o glutaraldeído por 20 minutos não foi suficiente para a desinfecção do $M$. tuberculosis e isso pode acarretar diagnósticos falso-positivos.

Neste trabalho, tanto na coloração para gram, quanto na cultura de aeróbios, o $\mathrm{G}$ de Cochran foi significativo nos frascos 1 e 4 .

A coloração para gram mostrou-se negativa no frasco 1 de todas as amostras, como era esperado, pois esse representa o broncoscópio antes de ser utilizado no pa- ciente, devendo estar, portanto, desinfetado. No entanto, houve um caso $(1,5 \%)$ de contaminação no frasco 4 , isto é, depois da desinfecção de alto nível.

Chamam a atenção na cultura de aeróbios os frascos 1 e 4, porque estes apresentaram crescimento de microorganismos em 9,2\% e 16,9\% das amostras. Nesses frascos não deveria haver crescimento, pois eles representam o broncoscópio, quando este deveria estar desinfetado, isto é, antes do exame e após a desinfecção de alto nível, respectivamente. Em suma, o broncoscópio não estava chegando desinfetado ao paciente e o processo de desinfeç̧ão não foi eficiente nesses casos. Devemos sempre ressaltar que a apropriada limpeza e desinfecção do broncoscópio são obrigatórios para prevenir infecções e pseudo-infecções.

Está, também, evidente a contaminação do broncoscópio pelas vias aéreas superiores, devido à presença de bactérias características da microbiota normal da boca $e$ fossas nasais, e de outras, presentes em pacientes que recebem antibioticoterapia, pois esta inibe a flora normal, permitindo que microorganismos como Klebsiella pneumoniae, Escherichia coli, Pseudomonas aeruginosa e Staphylococcus aureus cresçam em seu lugar ${ }^{(57,58)}$.

Chama-nos também a atenção a contaminação do broncoscópio por Staphylococcus epidermidis, presente na microbiota normal das mãos, o que pode representar má manipulação do aparelho após a sua desinfecção.

Quando comparamos a bacterioscopia com a bacteriologia, chama a atenção a presença de quatro casos de gram positivo e cultura negativa, que pode indicar que 0 microorganismo não estivesse mais hábil quando foi semeado.

Bactérias vegetativas são suscetíveis à ação do glutaraldeído. A 2\% é capaz de destruir muitas espécies vegetativas incluindo Staphylococus aureus, Proteus vulgaris, Escherichia coli e Pseudomonas aeruginosa em dois minutos. O glutaraldeído mostra-se como uma solução para os equipamentos que não podem ser esterilizados com método físico. Vários trabalhos demonstram a sua boa ação micobactericida ${ }^{(59)}$.

Leers $^{(27)}$, em 1980, recomenda tratamento com glutaraldeído a $2 \%$ por 10 a 30 minutos para desinfecção de endoscópios fibroópticos, precedido de adequada limpeza. Um grande número de trabalhos, também, demonstrou a atividade antiviral efetiva do glutaraldeído a $2 \%$, mesmo em presença de matéria orgânica, embora esta a lentifique $e^{(53,60)}$.

A matéria orgânica, inevitável e associada ao constante uso da solução, ajuda a mudar seu potencial germicida. Ela age protegendo o microorganismo ou competindo com ele pelos grupos dialdeídos livres. No entanto, a literatura indica que o glutaraldeído é resistente à presença de matéria orgânica. 
Ringrose ${ }^{(61)}$, Bassett(62) e Ayliffe e Deverill(63) associaram a resistência do microorganismo ao glutaraldeído ao tempo de contato menor que o recomendado para desinfecção.

Scheidt ${ }^{(64)}$, em 1980, encontrou contaminação de broncofibroscópios por Serratia e Pseudomonas, após desinfecção com glutaraldeído por 10 minutos. Somente depois de 45 minutos de imersão na solução foram encontradas as culturas negativas. Esse autor sugeriu que o tempo para esterilização fosse preferido ao tempo de desinfecção. Na rotina diária, isso é difícil, devido ao grande número de exames dos serviços de broncoscopia.

Spach et al. ${ }^{(40)}$, em um levantamento bibliográfico, referem ter encontrado 96 infecções transmitidas pelo broncoscópio, em que foram encontrados: Serratia marcescens, Pseudomonas aeruginosa, P. pseudomallei, M. tuberculosis, $M$. chelonae e avium.

Fraser et al. ${ }^{(65)}$ relatam $17 \%$ de cultura positiva após desinfecção do broncoscópio com glutaraldeído a $2 \%$. Já Suratt et al. ${ }^{(2)}$ não encontraram crescimentos de cultura de vários microorganismos após a desinfecção do broncoscópio.

$\mathrm{O}$ pH e a concentração também são determinantes da atividade do glutaraldeído. A solução do glutaraldeído é a $2 \%$, na qual foi adicionado um ativador que torna o $\mathrm{pH}$ próximo a 8 . Esse $\mathrm{pH}$ oferece um número ideal de dialdeídos livres, que são responsáveis pela atividade germicida desse desinfetante. Após 14 dias de uso contínuo a solução fica rediluída e o $\mathrm{pH}$ cai, diminuindo a atividade biocida.

Devido a seu arranjo complexo com canais e válvulas, o broncoscópio pode permanecer contaminado, a despeito de adequada limpeza e desinfecção.

Cortese e Prakash ${ }^{(45)}$ sugerem que estudos sejam feitos para avaliar a eficácia da limpeza e desinfecção dos componentes internos de endoscópios, inclusive separando os canais internos do equipamento para melhor avaliar a desinfecção.

Pappas et al.(66) relatam que em seu serviço, em Illinois, os broncoscópios eram limpos, enxaguados com água potável, desinfetados com glutaraldeído a $2 \%$ e novamente enxaguados com água potável. Após surto de infecções associadas à broncoscopia, com um óbito, os equipamentos começaram a ser esterilizados em óxido de etileno. Quando dois broncoscópios foram abertos para reparos, o canal de sucção de ambos estava danificado $e$ a porção do interior do broncoscópio, que era usualmente selada, estava cheia de matéria orgânica, em cuja cultura cresceram $M$. chelonei e Pseudomonas aeruginosa. Esse local não era atingido durante a limpeza $e$ desinfecção ou esterilização. Duas possibilidades foram levantadas pelos autores para a contaminação do broncoscópio: a água potável usada para o enxágüe ou o glu- taraldeído estavam contaminados. Isso talvez explique as culturas positivas encontradas no presente.

Segundo Rutala(24), esterilização é um termo absoluto e não relativo. Neste trabalho, tratamos com desinfecção de alto nível, que é o mínimo recomendado para garantir segurança aos pacientes submetidos à broncoscopia. Comparando os resultados obtidos com a literatura, concluímos que a desinfecção do broncoscópio em glutaraldeído a $2 \%$ por 20 minutos não foi eficiente em garantir a segurança de um procedimento invasivo sem riscos de contaminação aos pacientes, pois houve crescimento de Mycobacterium tuberculosis em 4,6\% dos frascos $1 \mathrm{e}$ $7,7 \%$ dos frascos 4 e de bactérias aeróbicas em 9,2\% e $16,9 \%$ dos frascos 1 e 4 , respectivamente.

Ficou evidente, também, a contaminação pela manipulação após a desinfecção, devido à presença de $S$. epidermidis no frasco 4 de algumas amostras (casos 3, $9 e$ 15).

\section{REFERÊNCIAS}

1. Prakash UBS. Pseudoepidemics of infections caused by bronchoscopy. Journal of Bronchology 1998;5:43-8.

2. Suratt PM, Smiddy JF, Gruber B. Deaths and complications associated with fiberoptic bronchoscopy. Chest 1976;69:747-51.

3. Rutala WA. Disinfection and sterilization of patient-care items. Infect Control Hosp Epidemiol 1996;17:377-84.

4. Costa MLM. Levantamento de rotinas de reprocessamento de endoscópio gastrintestinal em hospitais do município de São Paulo, 1996 (Tese de Mestrado, Escola Paulista de Medicina).

5. Pereira W Jr, Kovnat DM, Khan MA, Lacovino JR, Spivack ML, Snider GL. Fever and pneumonia after flexible fiberoptic bronchoscopy. Am Rev Respir Dis 1975;112:59-64.

6. Berman SO. Bronchoscopy and bacteremia. J Thorac Cardiovasc Surg 1960;40:635-9.

7. Burns DM, Shure D, Francoz R, Kalafer M, Harrell J, Witztum K, Moser KM. The physiologic consequences of saline lobar lavage in healthy human adults. Am Rev Respir Dis 1983;127:695-701.

8. Credle WF Jr, Smiddy JF, Elliot RC. Complications of fiberoptic bronchoscopy. Am Rev Respir Dis 1974;109:67-72.

9. Pereira W Jr, Kovnat DM, Khan MA, Lacovino JR, Spivack ML, Snider GL. Fever and pneumonia following fiberoptic bronchoscopy [abstract]. Am Rev Respir Dis 1974;109:692.

10. Pingleton SK, Harrison GF, Stechschulte DJ, Wesselius LJ, Kerby GR, Ruth WE. Effect of location, $\mathrm{pH}$ and temperature of instilate in bronchoalveolar lavage in normal volunteers. Am Rev Respir Dis 1983; 128:1035-7.

11. Pugin J, Suter PM. Diagnostic bronchoalveolar lavage in patients with pneumonia produces sepsis like systemic effects. Intensive Care Med 1992;18:6-10.

12. Titche LL. Postbronchoscopic reactions. Ann Otol 1945;54:568-9.

13. Van Gundy K, Boylen CT. Fiberoptic bronchoscopy. Indications, complications, contraindications. Postgrad Med 1988;83:289-94,

14. Witte MC, Opal SM, Gilbert JG, Pluss JL, Thomas DA, Olsen JD, et al. Incidence of fever and bacteremia following transbronchial needle aspiration. Chest 1986;89:85-7.

15. Standiford TJ, Kunkel SL, Strieter RM. Elevated serum levels of tumor necrosis factor alpha after bronchoscopy and bronchoalveolar lavage. Chest 1991;99:1529-30.

16. Beyt BE Jr, King DK, Glew RH. Fatal pneumonitis and septicemia after fiberoptic bronchoscopy. Chest 1977;72:105-7. 
17. Hsu JT, Barrett CR Jr. Lung abscess complicating transbronchial biopsy of a mass lesion. Chest 1981;80:230-2.

18. Hammer DL, Aranda CP, Galati V, Adams FV. Massive intrabronchial aspiration of contents of pulmonary abscess after fiberoptic bronchoscopy. Chest 1978;74:306-7.

19. Aelony Y, Finegold SM. Serious infectious complications after flexible fiberoptic bronchoscopy. West J Med 1979;131:328-33.

20. Radner DD. Post bronchoscopic reactions in pulmonary tuberculosis. Ann Rev Tuberc 1943;47:370-8.

21. Rimmer J, Gibson P, Bryant DH. Extension of pulmonary tuberculosis after fiberoptic bronchoscopy. Tubercle 1988;69:57-61.

22. Sesma P, Sanchez F, Deben FM. Tuberculous pneumonia: a complication of bronchoscopy. Rev Clin Esp 1984;173:185-6.

23. Hanson PJV, Gor D, Clarke JR, Chadwick MV, Gazzard B, Jeffries DJ, et al. Recovery of the human immunodeficiency virus from fiberoptic bronchoscopes. Thorax 1991;46:410-2.

24. Rutala WA. Draft APIC guideline for selection and use of disinfectants. Am J Infect Control 1995;23:35A-67A.

25. Oho K, Amemiya R. Practical fiberoptic bronchoscopy. Tokyo: IgakuShoin, 1980.

26. Dawson DJ, Armstrong JG, Blacklock ZM. Mycobacterial cross-contamination of bronchoscopy specimens. Am Rev Respir Dis 1982; 126:1095-7.

27. Leers WD. Disinfection endoscopies: how not to transmit Mycobacterium tuberculosis by bronchoscopy. Can Med Assoc J 1980;123:27580.

28. Brasil - Portaria № 15, de 27 de agosto de 1988. Expede normas, na forma de anexo, para registro dos saneantes domissanitários com ação antimicrobiana. Diário Oficial da União, Brasília, p. 17.041, 5 set. 1988. Seção 1.

29. Brasil - Portaria № 930, de 27 de agosto de 1992. Expede normas, na forma de anexos, para o controle de infecções hospitalares. Diário Oficial da União, Brasília, p. 1279, 4 set. 1992. Seção 1.

30. Brasil - Ministério da Saúde. Coordenação de Controle de Infecção Hospitalar. Processamento de Artigos e Superfícies em Estabelecimento de Saúde. Brasília, 1993, p. 15.

31. Centers for Disease Control and Prevention - Recommendations for preventing transmission of human immunodeficiency virus and hepatitis $\mathrm{B}$ virus to patients during exposure-prone invasive procedures. MMWR 1991;40(RR-8):1-9.

32. Centers for Disease Control and Prevention - Nosocomial infection and pseudo-infection from contaminated endoscopies and bronchoscopes - Wisconsin and Missouri. MMWR 1991;40:675-8.

33. Centers for Disease Control and Prevention - Prevention and control of tuberculosis in US communities with risk minority populations and prevention and control of tuberculosis among homeless persons: recommendations of the Advisory Council for Elimination of Tuberculosis. Morb Mortal Wkly Rep 1992;41:1-23.

34. Centers for Disease Control and Prevention - Draft guideline for isolation precautions in hospitals. Federal Register 1994;59:55552-70.

35. São Paulo (Estado). Secretaria da Saúde. Centro de Apoio ao Desenvolvimento de Assistência Integral à Saúde (CADAIS) - Organização do Centro de Material e Noções de Esterilização. São Paulo, 1993, p. 36-41 (Cadernos de Saúde).

36. Kaczmarek RG, Moore RM. Multi-state investigation for the actual disinfection and sterilization of endoscopies in health care facilities. Am J Med 1991;92:257-61.

37. Mayhall CG. Hospital epidemiology and infection control. Williams \& Wilkins, 1996;47:680-93.

38. Mehta AC, Curtis PS. The high price of bronchoscopy. Maintenance and repair of flexible fiberoptic bronchoscope. Chest 1990;98:448 54.

39. Rodrigues E. Infecções hospitalares: prevenção e controle. São Paulo: Sarvier, 1997;634.

40. Spach DH, Silverstein FE, Stamm WE. Infections transmitted by gastrintestinal endoscopy and bronchoscopy. Ann Intern Med 1993;118: 117-28.
41. Strange C, Barbarash RA, Heffner JE. Lidocaine concentrations in bronchoscopic specimens. Chest 1988;93:547-9.

42. Wimberley N, Willey S, Sullivan N, Bartlett JG. Antibacterial properties of lidocaine. Chest 1979;76:37-40.

43. NCCLS (National Committee for Clinical Laboratory Standard), M100S9, 1999.

44. Siegel S, Castellan Jr NJ. Nonparametric Statistics. $2^{\text {nd }}$ ed. New York: McGraw-Hill Int. Ed., 1988;399p.

45. Cortese DA, Prakash UBS. Bronchoscopy in pulmonary infections. In Prakash UBS, editor. Bronchoscopy. Mayo Foundation. New York: Raven Press Ltd., 1994;183-94.

46. Rutala WA. FDA Labeling requirements for disinfection of endoscopes: a counterpoint. Infect Control Hosp Epidemiol 1995;16:231-5.

47. Penco JCF, Martin MA. Chemical germicide treatment of flexible endoscopes: the risk of infection transmission from errors in disinfection practices. In: Rutala WA, editor. Chemical germicides in health care International symposium. APIC. Washington DC, 1995;61-71.

48. Pepper RE, Lieberman ER. Dialdehyde alcoholic sporicidal compositions. US Patent No. 1962;3,016,328.

49. Stonehill AA, Krop S, Borick PM. Buffered glutaraldehyde - A new chemical sterilizant solution. Am J Hosp Pharmacol 1963;20:458-65.

50. Spaulding EH. Chemical disinfection of medical and surgical materials. In: Lawrence CA, Block SS, editors. Disinfection, sterilization and preservation. Philadelphia, PA: Lea \& Febiger, 1968;517-31.

51. Borick PM, Dondershine FH, Chandler JL. Alkalinized glutaraldehyde, a new antimicrobial agent. J Pharm Sci 1964;53:1273-5.

52. Rubbo SD, Gardner JF, Webb RL. Biocidal activities of glutaraldehyde and related compounds. J Appl Bacteriol 1967;30:78-87.

53. Sammartino MT, Israel RH, Magnussen CR. Pseudomonas aeruginosa contamination of fiberoptic bronchoscopes. J Hosp Infect 1982;3:65 71.

54. Baughman RP, Dohn MN, Loudon RG, Frame PT. Bronchoscopy with bronchoalveolar lavage in tuberculosis and fungal infections. Chest 1991;99:92-7.

55. Davis D, Bonekat HW, Andrews D, Shigeoka JW. Disinfection of the flexible fiberoptic bronchoscope against Mycobacterium tuberculosis and M. gordonae. Thorax 1984;39:785-8.

56. Carson LA, Petersen NJ, Favero MS. Growth characteristics of atypical mycobacteria in water and their comparative resistance to disinfectants. Appl Environ Microbiol 1978;36:839-46.

57. Nelson KE, Larson PA, Schraufnagel DE, Jackson J. Transmission of tuberculosis by flexible fiberbronchoscope. Am Rev Respir Dis 1983; 127:97-100

58. Wanner A, Amikam B, Robinson MJ, Anandam EJ, Sackner MA. Comparison between the bacteriologic flora of different segments of the airways. Respiration 1973;30:561-9.

59. Scott EM, Gorman SP. Glutaraldehyde. In: Block SS, editor. Disinfection, sterilization and preservation. $4^{\text {th }}$ ed. Philadelphia: Lea, 1991 596-614.

60. Borick PM, Pepper RE. The spore problem. In: Bernarde MA, editor. Disinfection. New York: Marcel Dekker Inc., 1970;85-102.

61. Ringrose RE. A hospital outbreak of Serratia marcescens associated with ultrasonic nebulizers. Ann Intern Med 1968;69:719-29.

62. Bassett DJC. Common-source outbreaks. Proc R Soc Med 1971;64: 18-24.

63. Ayliffe GA, Deverill CEA. Decontamination of gastroscopes. Health Soc Serv J 1979; 538-40.

64. Scheidt A. Persistent contamination of the flexible fiber-bronchoscope following disinfection in aqueous glutaraldehyde. Chest 1980;78:352 2 .

65. Fraser V, O'Rourke S, Jones M, Murray P, Clouse RE, Klasner J, et al. Gastrointestinal endoscope disinfection: a prospective randomized trial comparing automated and manual disinfection [Abstract 12]. Gastrointest Endosc 1992;38:277.

66. Pappas SA, Schaaff DM, DiConstanzo MB, King Jr FW, Sharp JT. Contamination of flexible bronchoscopes. Am Rev Respir Dis 1983 127:391-2. 
ANEXO

Comparação dos resultados de coloração para gram e cultura de aeróbios

\begin{tabular}{|c|c|c|c|c|c|c|c|c|}
\hline Amost. & Fr. 1 Gram & Fr. 1 Cultura & Fr. 2 Gram & Fr. 2 Cultura & Fr. 3 Gram & Fr. 3 Cultura & Fr. 4 Gram & Fr. Cultura \\
\hline 1 & - & - & + & SV/SA & - & SV/S A & - & - \\
\hline 2 & - & - & + & SV & - & SV & - & - \\
\hline 3 & - & - & + & SV/EC/Esp & + & $\mathrm{SV} / \mathrm{KP}$ & - & SE \\
\hline 4 & - & - & + & SV & - & SV & - & - \\
\hline 5 & - & - & - & SV/S A & - & SV & - & - \\
\hline 6 & - & - & - & C sp & - & C sp & - & - \\
\hline 7 & - & - & + & $\mathrm{S} A / \mathrm{S} V$ & - & $S V^{\prime}$ & - & - \\
\hline 8 & - & SV & + & - & + & $\mathrm{Esp} / \mathrm{ABh}$ & + & - \\
\hline 9 & - & - & - & SV & - & SV/SE & - & SV/SE \\
\hline 10 & - & - & - & SV & - & SV & - & - \\
\hline 11 & - & - & - & SV/Esp & - & SV/Esp & - & - \\
\hline 12 & - & - & + & $\mathrm{S} A / \mathrm{SV}$ & + & PA & - & - \\
\hline 13 & - & - & + & $\mathrm{SA} / \mathrm{KP}$ & - & PA & - & - \\
\hline 14 & - & - & - & Ec sp & - & SV & - & - \\
\hline 15 & - & - & - & SV & - & SE/PA & - & SE \\
\hline 16 & - & - & + & SV & - & SP & - & - \\
\hline 17 & - & - & - & SV/S A & - & $\mathrm{PA} / \mathrm{S} \mathrm{A}$ & - & S A \\
\hline 18 & - & - & - & SV/AB h & - & SV & - & - \\
\hline 19 & - & - & - & PA & - & $\mathrm{PA} / \mathrm{SP}$ & - & - \\
\hline 20 & - & - & - & $\mathrm{EC}$ & _- & EC & - & - \\
\hline 21 & - & - & - & SV & - & SV & - & - \\
\hline 22 & - & - & + & - & + & Esp & - & - \\
\hline 23 & - & - & - & $\mathrm{S} A / \mathrm{SV}$ & - & SA & - & - \\
\hline 24 & - & - & + & PA & - & $\mathrm{SA} A / \mathrm{AB} h$ & - & PA \\
\hline 25 & - & - & - & SV & - & SE & - & - \\
\hline 26 & - & - & - & SV & - & $\mathrm{Esp} / \mathrm{KP}$ & - & - \\
\hline 27 & - & - & + & $\mathrm{KP}$ & - & $\mathrm{KP}$ & - & Esp \\
\hline 28 & - & - & - & $\mathrm{AB} \mathrm{h}$ & - & $\mathrm{ABh}$ & - & - \\
\hline 29 & - & - & - & SV & - & Stenotrop. & - & - \\
\hline 30 & - & - & - & $\mathrm{KP}$ & - & $\mathrm{KP}$ & - & - \\
\hline 31 & - & - & + & - & + & Esp & - & - \\
\hline 32 & - & - & - & SBH-A & - & SBH-A & - & SBH-A \\
\hline 33 & - & - & - & SV & - & - & - & - \\
\hline 34 & - & - & - & CBsp/SV & - & - & - & - \\
\hline 35 & - & - & - & PA & - & PA & - & - \\
\hline 36 & - & SCneg. & - & SV & - & $\mathrm{PA}$ & - & - \\
\hline 37 & - & - & - & SV/S A & - & S A/SV & - & - \\
\hline 38 & - & - & - & PA & - & PA & - & - \\
\hline 39 & - & - & + & PA & _- & PA & - & - \\
\hline 40 & - & - & - & SV/SC neg & - & PR & - & - \\
\hline 41 & - & - & - & Ec sp & - & PA & - & - \\
\hline 42 & - & - & - & PA/KP & - & PA & - & - \\
\hline 43 & - & - & - & $\mathrm{PA} / \mathrm{SV}$ & - & SV & - & - \\
\hline 44 & - & - & + & SCneg/SV & - & SCneg/SV & - & - \\
\hline 45 & - & - & - & SV & - & $\mathrm{EC} / \mathrm{PA}$ & - & SCneg. \\
\hline 46 & - & SCneg. & + & $\mathrm{PM} / \mathrm{KP}$ & + & $\mathrm{PA} / \mathrm{Ec} \mathrm{sp}$ & - & - \\
\hline 47 & - & - & - & SCneg/SV & - & $\mathrm{SV} / \mathrm{PA}$ & - & - \\
\hline 48 & - & - & + & $S A$ & - & SV/PA/Ec sp & - & - \\
\hline 49 & - & - & + & $\mathrm{KP}$ & + & $\mathrm{PA} / \mathrm{KP}$ & - & - \\
\hline 50 & - & - & + & S A/SV & + & $\mathrm{KP} / \mathrm{PA}$ & - & - \\
\hline 51 & - & - & - & SCneg/SV & - & KP & - & - \\
\hline 52 & - & - & - & SCneg & - & $\mathrm{KP} / \mathrm{PA}$ & - & - \\
\hline 53 & - & - & - & SV/KP & + & SV/KP & - & - \\
\hline 54 & - & - & - & SV & - & Ec sp/PA & - & SCneg \\
\hline 55 & - & SCneg. & - & Ec sp/SCneg & - & $\mathrm{S}$ A/PA & - & - \\
\hline 56 & - & - & + & S A & + & Ec sp/S A/AB & - & SCneg \\
\hline 57 & - & - & + & $\mathrm{PA} / \mathrm{Esp}$ & - & Esp & - & - \\
\hline 58 & - & - & - & SV & - & SV & - & SCneg \\
\hline 59 & - & SCneg & - & Esp/SBH-B & + & Esp/SBH-B & - & - \\
\hline 60 & - & - & - & $\mathrm{S} A / \mathrm{SV}$ & - & SA & - & - \\
\hline 61 & - & - & + & SV & _- & SV/SCneg & - & - \\
\hline 62 & - & SCneg & - & SCneg & + & Esp/PA/AB & - & - \\
\hline 63 & - & - & - & SCneg & + & $\mathrm{PA} / \mathrm{Esp}$ & - & - \\
\hline 64 & - & - & + & SCneg/SV & - & SCneg/SV & - & - \\
\hline 65 & - & - & - & $K P$ & - & PR/SV & - & - \\
\hline
\end{tabular}

Stenotrop - Stenotrophomonas; Esp - Enterobacter sp; PA - Pseudomonas aeruginosa; SP - Streptoc. pneumoniae; PR - Providencia rettgeri; Csp - Corinebacterium sp; Cbsp Citrobacter sp; EC sp - Enterococcus sp; SV - Streptococcus viridans; Kp - Klebsiella pneumoniae; Scneg - Staphylococcus coagulase neg.; SBH-B - S. hemoliticus B; S A - Staphylococcus aureus; SBH-A - S. hemoliticus A; PM - Proteus mirabilis; EC - Escherichia coli; AB.h - Acinetobacter baum. hae.; SE - Staphylococcus. 